Chapter 11

\title{
Self-Coherent Reflective Passive Optical Networks
}

\author{
S. Straullu, S. Abrate and R. Gaudino \\ Additional information is available at the end of the chapter \\ http://dx.doi.org/10.5772/58999
}

\section{Introduction}

Fiber optic access networks are the necessary infrastructural approach for a real broadband delivery, allowing the fiber to arrive closer to the final customer, eventually up to the premises equipment; such infrastructures, depending on the depth of reach of the fiber, are usually referred to as FTTX (Fiber To The X), where X stands for H (Home), B (Building), C (Curb) or $\mathrm{Cab}$ (Cabinet). They are the basis for broadband access networks, enabling high-speed Internet access, digital TV broadcast (IPTV), video on demand (VOD) and other services. Comparing with copper technologies, like for example DSL (digital subscriber line), higher bandwidths (up to several $10 \mathrm{Gbit} / \mathrm{s}$ ) and higher distances (up to several tens of $\mathrm{km}$ ) are possible. FTTX infrastructures can address both residential and business access, and support mobile backhauling.

Access networks can be divided in two main categories: Active Optical Networks (AONs) and Passive Optical Networks (PONs):

- AONs are a point-to-point (P2P) network structure, where all users have their own fiber optic line that is terminated on an optical access node, which can be designed differently depending on specifications;

- PONs feature a point-to-multi-point (P2MP) tree-like architecture (as shown in Fig. 1 below) to provide broadband access, based on a network composed by an Optical Line Terminal (OLT) at the service provider's Central Office (CO) and several Optical Network Units (ONUs) at the users' premises. PONs allow only passive elements along the Optical Distribution Network (ODN) that connects the OLT to the ONUs. The main advantages are that PONs do not require electrical power in the outside plant to power the distribution elements, thereby lowering operational costs and complexity and, moreover, they allow to highly reduce the number of required optical ports in the CO compared to P2P solutions. 
The OLT is the interface between backbone and access networks, and it is also responsible for the enforcement of any media access control (MAC) protocol for upstream bandwidth arbitration. The ONU cooperates with the OLT in order to control and monitor all PON transmission and to enforce the MAC protocol for upstream bandwidth arbitration; it also acts as the residential gateway, coupling the ODN with the in-home network.

The ODN consists of the distribution fibers and all the passive optical distribution elements, mainly optical splitters and/or wavelength-selective elements (WDM filters), that are located in sockets or cabinets. The splitting ratio in most cases is between 1:8 and 1:128, and can be performed in lumped or cascaded elements. The ODN power budget is usually taken as a reference for PON reach calculations, and is computed as the difference between the transceiver back-to-back power budget (i.e. OLT transmitter directly coupled into ONU receiver or vice versa) and the passive optical equipment necessary inside the OLT and the ONU to perform all multiplexing of PON signals into a single fiber. Hence, the ODN power budget considers the remaining power budget that can be spent for the distribution fibers and the distribution elements in the remote nodes.

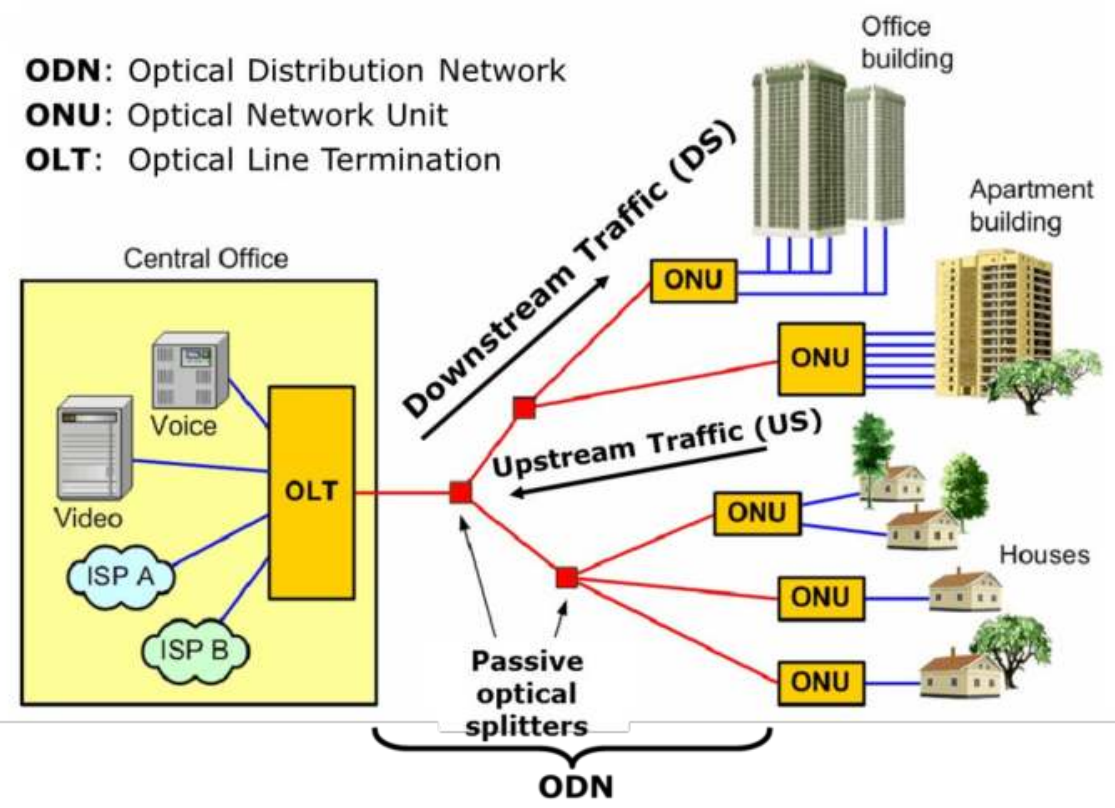

Figure 1. Passive Optical Network architecture

During the last 20 years, the Full Service Access Network (FSAN) and the Ethernet in the First Mile alliance (EFM) working groups, in cooperation with the International Telecommunications Union (ITU) and the Institute of Electrical and Electronics Engineers (IEEE) standardization bodies, defined several PON standards, as summarized in Figure 2. 
For the purposes of this Chapter, we will not go through all these standards, since none of these has been approached with self-coherent reflective architectures that will be presented in this Chapter, but we will mention only the most recent one, called NG-PON2 (Next Generation PON 2), in progress of standardization at the time of writing and with still open technological issues. In particular, we will address only physical layer aspects. The definition of such standard started in 2010 by FSAN.

Since about the $70 \%$ of the total investments in deploying PONs is due to the optical distribution networks, it is crucial for the NG-PON2 evolution to be compatible with the deployed networks and to reuse the outside plant. Moreover, NG-PON2 technology must outperform existing PON technologies in terms of ODN compatibility, bandwidth, capacity and costefficiency. For this reason, the initial "wish list" for NG-PON2 was originally very demanding, requiring not only an increase of bit rates with respect to previous generations, but also in terms of total number of users per PON tree and reach.

The major original general requirements of NG-PON2 can be summarized as follows:

- increase the aggregate rate to $40 \mathrm{Gbit} / \mathrm{s}$ in downstream or upstream;

- increase the reach to $40-60 \mathrm{~km}$ (or more);

- increase the splitting ratio up to 256 users (or more);

- compatibility with the attenuation classes defined in XG-PON, as reported in Table 1;

- increase the effective bit rate per user (in both directions) to a value closer to $1 \mathrm{Gbit} / \mathrm{s}$ per ONU.

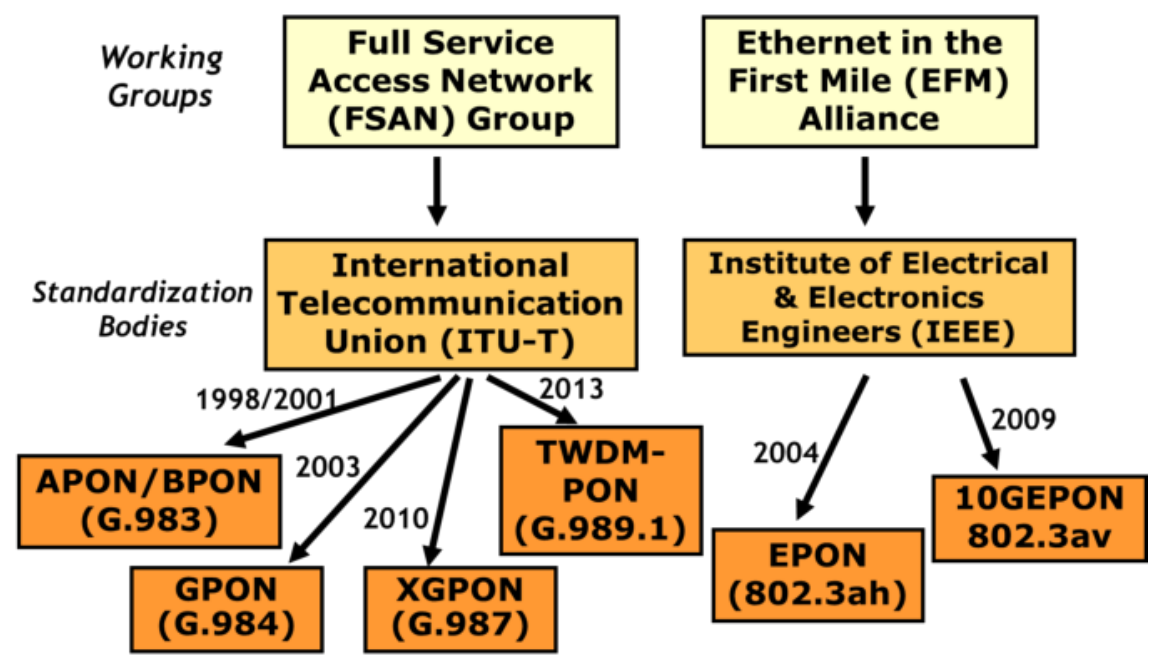

Figure 2. PON standards 


\begin{tabular}{ccccc}
\hline & $\begin{array}{c}\text { 'Nominal1' class (N1 } \\
\text { class) }\end{array}$ & $\begin{array}{c}\text { 'Nominal2' class (N2 } \\
\text { class) }\end{array}$ & $\begin{array}{c}\text { 'Extended1' class (E1 'Extended2' class (E2 } \\
\text { class) }\end{array}$ & \begin{tabular}{c} 
class) \\
\hline Minimum loss
\end{tabular} \\
\hline $14 \mathrm{~dB}$ & $16 \mathrm{~dB}$ & $18 \mathrm{~dB}$ & $20 \mathrm{~dB}$ \\
\hline Maximum loss & $29 \mathrm{~dB}$ & $31 \mathrm{~dB}$ & $33 \mathrm{~dB}$ & $35 \mathrm{~dB}$ \\
\hline
\end{tabular}

Table taken from G.987.2

Table 1. Classes for optical XG-PON path loss

Many PON technologies have been proposed to provide broadband optical access beyond 10 Gbit/s, like for example:

- the 40 Gigabit time-division multiplexed PON (XLG-PON) proposal [1] which increases the single carrier serial downstream bit rate of a 10 Gigabit PON (XG-PON) to 40 Gbit/s;

- a set of orthogonal frequency-division multiplexed (OFDM)-based PON proposals which employ quadrature amplitude modulation and fast Fourier transform to generate digital OFDM signals for transmission [2, 3];

- a group of WDM-PON proposals which provide a dedicated wavelength channel at the rate of $1 \mathrm{Gbit} / \mathrm{s}$ to each ONU with different WDM transmit or receive technologies [4, 5];

- the time-and wavelength-division multiplexed PON (TWDM-PON) proposal which stacks multiple XG-PONs using WDM [6].

The first proposal, based on a single wavelength (per direction) at very high bit rate, was finally discarded, since it was perceived as too expensive. Indeed, it had to include a duo-binary modulation and a downstream transmission around $1300 \mathrm{~nm}$ to avoid dispersion compensation. Moreover, it was not scalable, thus no future upgrades above $40 \mathrm{Gbit} / \mathrm{s}$ downstream seemed feasible on a single wavelength.

The proposals based on OFDM are very interesting and technologically advanced solutions, but they do not easily guarantee a complete compatibility with existing deployed networks and devices.

Regarding the WDM-PON architectures, a key point is whether the WDM filters are inside the ODN or in the ONU.

The "pure" WDM-PON approach, based on WDM filters inside the ODN, generates some constraints to Telecom operators:

- all passive splitters installed in the already deployed PON (brown field scenario) need to be substituted with Arrayed Waveguide Grating (AWG) filters;

- limited backward compatibility with already deployed standards (GPON, EPON, XG-PON, etc.);

- a dedicated wavelength per user is a "circuit" per individual user, thus no sharing or statistical multiplexing is possible. 
For these reasons, this solution seems to be out of question for NG-PON2 standard. Among all of the aforementioned proposals, TWDM-PON has attracted the majority support from global vendors and was selected by the FSAN community in the April 2012 meeting as a primary solution to NG-PON2. TWDM-PON increases the aggregate PON rate by stacking XG-PONs via multiple pairs of wavelengths; since an XG-PON system offers the access rates of $10 \mathrm{Gbit} / \mathrm{s}$ in downstream and $2.5 \mathrm{Gbit} / \mathrm{s}$ in upstream, a TWDM-PON system with four pairs of wavelengths will then be characterized by:

- 4 wavelengths (upgradable to 8) at $100 \mathrm{GHz}$ WDM spacing:

- $1595-1600 \mathrm{~nm}$ for downstream (up to $1605 \mathrm{~nm}$ with 8 wavelengths);

- $1535-1540 \mathrm{~nm}$ for upstream (up to $1545 \mathrm{~nm}$ with 8 wavelengths);

- $40 \mathrm{Gbit} / \mathrm{s}$ in downstream (10 Gbit/s per wavelength) and $10 \mathrm{Gbit} / \mathrm{s}$ in upstream (2.5 Gbit/s per wavelength);

- up to 64 ONUs (16 per wavelength);

- up to $40 \mathrm{~km}$;

- up to $35 \mathrm{~dB}$ of power budget;

- full backward compatibility.

The basic TWDM-PON architecture is shown in Figure 3. Four XG-PONs are stacked by using four pairs of wavelengths (e.g., wavelength pairs of $\{\lambda 1, \lambda 5\},\{\lambda 2, \lambda 6\},\{\lambda 3, \lambda 7\}$ and $\{\lambda 4, \lambda 8\}$, in Figure 3). ONUs are equipped with tunable transmitters and receivers. The tunable transmitter is tunable to any of the four upstream wavelengths; the receiver is tunable to any of the four downstream wavelengths. In order to achieve power budget higher than that of XG-PON, optical amplifiers (OAs) are used at the OLT side to boost the downstream signals as well as to pre-amplify the upstream signals. The ODN remains passive since OAs are placed at the OLT side, together with WDM Mux/DeMux.

Therefore, the TDWM-PON key features are:

- colorless ONUs;

- splitter-based ODN.

Most of the TWDM-PON components are commercially available in access networks today. Comparing to previous generations of PONs (e.g., GPON, XG-PON), the only, but technically very challenging, new components in TWDM-PON are the tunable receivers and tunable transmitters at the ONU.

For what concerns the tunable receiver, it should tune its wavelength to any of the TWDMPON downstream wavelengths by following the OLT commands. This function can be implemented by using candidate technologies such as:

- thermally tuned Fabry-Perot (FP) filter [7];

- angle-tuned FP filter, injection-tuned silicon ring resonator [8]; 


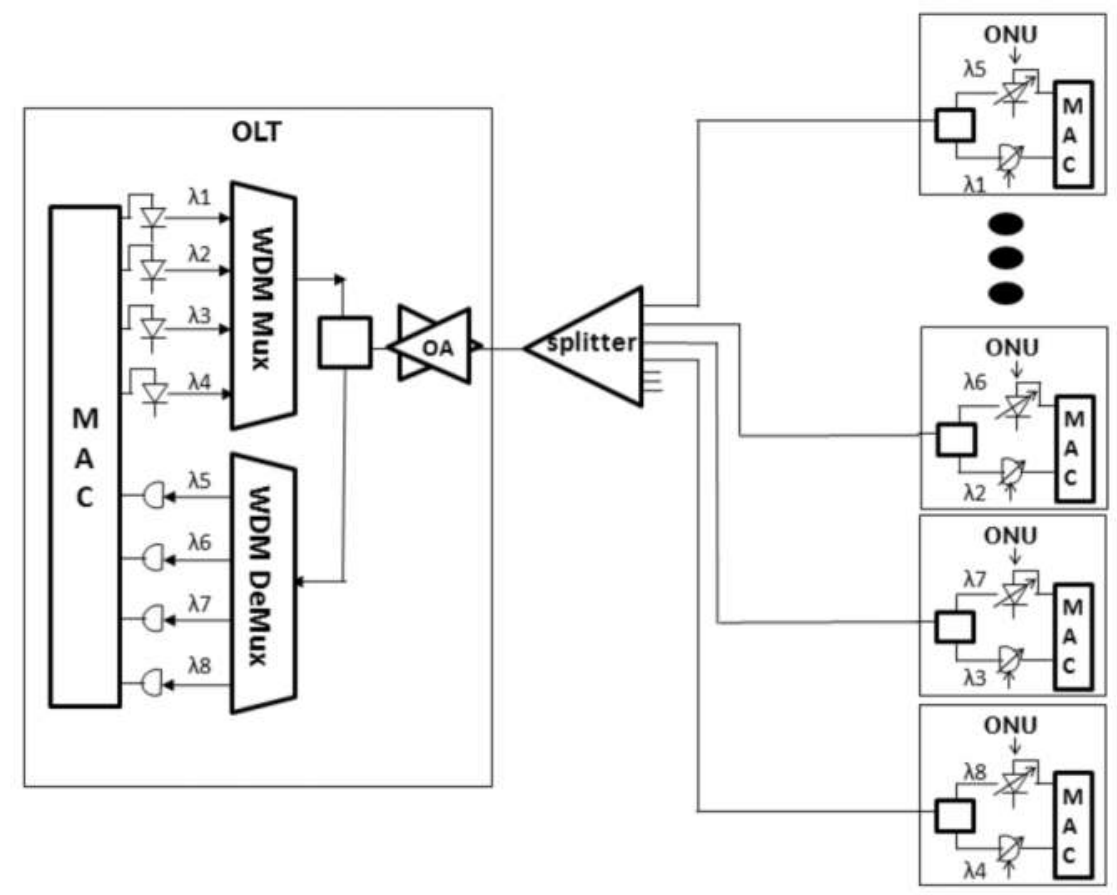

$\overline{\text { Picture taken from }}[6]$

Figure 3. TWDM-PON system diagram

- liquid crystal tunable filter [9];

- thermally tunable FP detector [10].

The ONU tunable transmitter can tune its wavelength to any of the upstream wavelengths. The related implementation technologies are:

- distributed feedback (DFB) laser with temperature control (TC) [11];

- DFB laser with partial TC [12];

- multi-section distributed Bragg reflector laser (electrical control) without cooling [13];

- external cavity laser (ECL) with mechanical control without cooling [14];

- ECL with thermo/electro/piezo/magneto-optic control without cooling [15, 16].

All these solutions are today under consideration for NG-PON2, but none has yet completely demonstrated to be achievable at the (very low) target prices for ONU. Purpose of this Chapter is to describe a solution for the upstream transmission that avoids the need for a tunable laser at the ONU side: it is based on self-coherent reflective PON architectures as a possible 
technological approach to the NG-PON2 requirements. In the following we will then first show the concept of the reflective PON, describing the key components it needs, the problems it solves and the limitations it encounters; then, we will propose the self-coherent detection enhancement and will give an overview of the latest research results available in literature.

\section{The reflective PON approach}

A very basic schematic view of a WDM-PON architecture is depicted in Figure 4. It makes use of integrated multichannel transmit (TX) and receive (RX) arrays in the OLT, and tunable laser (T-TX) and filters (TOF) in the ONUs. From the OLT, all downstream wavelengths are broadcast via the ODN; therefore, a tunable filter is necessary at each ONU in order to select the correct wavelength. Similarly, each ONU has to be provided with a tunable laser for the correct upstream wavelength selection.

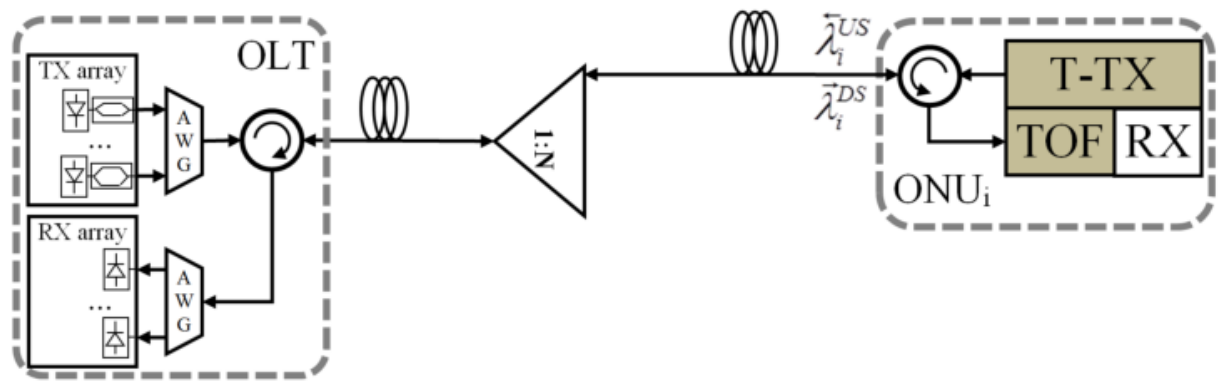

Figure 4. WDM-PON architecture

One of the few physical layer problems over which FSAN is still working today before the final NG-PON2 Recommendation release, is related to these two devices at the ONU. Indeed, they should both have a precision compatible with a $100 \mathrm{GHz}$ wavelength grid, and be able to tune on (at least) four wavelengths, adopting some protection systems during their switch-on time, in order to avoid interferences with other channels (due to the uncontrolled wavelength transmission at the laser power on); moreover, they should operate on a very wide temperature range and they should have a target price compatible with equipment to be installed at the customer premises. Moreover, in the longer term, if more than four wavelengths will be used (for instance for WDM overly), this issue will be particularly critical.

An alternative solution to overcome this problem is represented by reflective PON architectures, whose key idea is to generate the unmodulated upstream wavelengths at the OLT side, and modulate them in reflection at the ONU side, as schematically depicted in Figure 5. With respect to the setup depicted in Figure 4, the OLT is equipped by an additional multichannel transmit array, for the unmodulated seed signals generation, and the tunable laser at the ONU side is replaced by a reflective transmitter (R-TX). This way, the costs and the technical issues 
(the wavelength control in particular) related to the tunable lasers are moved from the ONU to the OLT, where they can be more easily managed.

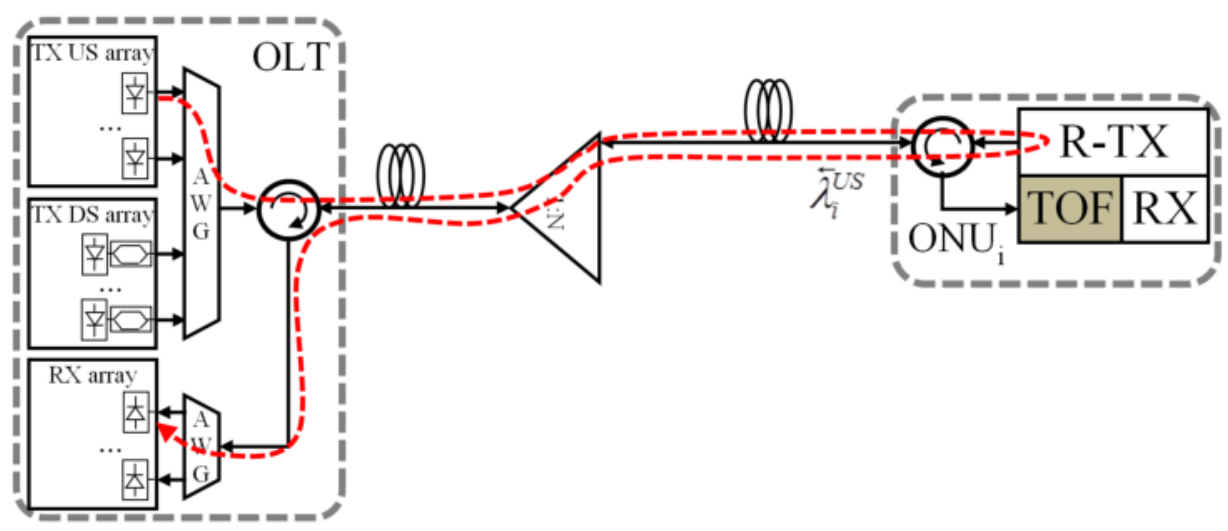

Figure 5. Scheme of a possible solution for Reflective-PON ONUs

\subsection{Key components for the reflective ONU}

Many variants of WDM reflective PON architectures can be found in literature, all around the common denominator of avoiding expensive tunable lasers at the ONU by means of using a reflective modulator for the upstream transmission that sends back a centrally-generated seed signal. One of the most common approaches adopted in order to obtain this effect at the ONU side is based on the use of Reflective Semiconductor Optical Amplifier (RSOA), which combine the amplification and modulation capabilities in a single device. They are composed by a high reflectivity coating on one facet, along with a curved waveguide and ultra-low reflectivity coating on the other facet, to produce a highly versatile reflective gain medium [17]. Today, few optical devices manufacturers propose commercial RSOA devices (e.g. as CIP, MEL and Kamelian), since they are just used in the reflective PON scenario and at a research level.

Figure 6 schematically depicts a RSOA in a typical low-cost TO-CAN package, while Figure 7 shows the electrical bandwidth measurement result of a typical commercial RSOA (butterflypackaged solutions may have higher bandwidth, but their cost seems too high for application in the extremely cost-sensitive scenario of $\mathrm{ONU}$ ). From this graph, it is possible to notice that the $3 \mathrm{~dB}$ electrical bandwidth of the device under test is about $500 \mathrm{MHz}$; anyway, the signal obtained by modulating the RSOA bias current $I_{b}$ with a $1 \mathrm{Gbit} / \mathrm{s}$ OOK modulation (shown in the inset of Figure 7) is received without inter-symbol interference.

Since 2000, the interest of RSOA as upstream transmitters for WDM-PON applications based on reflective ONU has grown-up. The first results have been proposed in [18], where the uplink data stream was reflected and modulated via the RSOA at $1.25 \mathrm{Gbit} / \mathrm{s}$, as then further developed and investigated in several later works (e.g. [19-22]). 


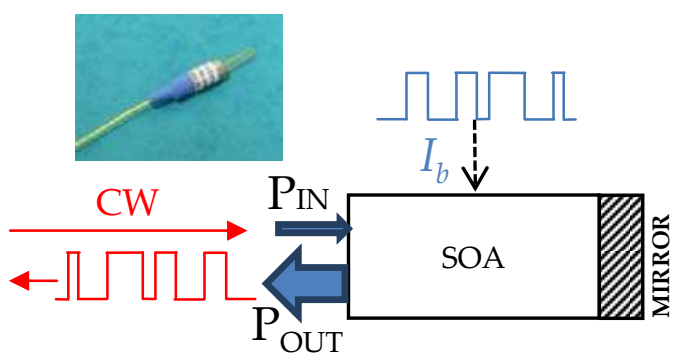

Figure 6. Schematic view of a RSOA

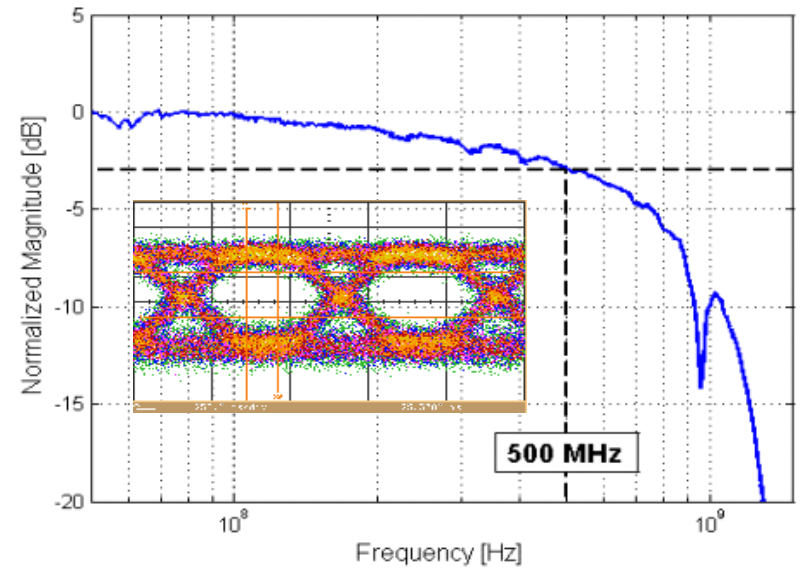

Figure 7. Characterization of a RSOA

In order to increase the upstream data rate up to $10 \mathrm{Gbit} / \mathrm{s}$ in such an architecture, using this low-bandwidth devices, different approaches have been proposed by research groups, like the use of optical filtering and electronic equalization [23-25] or the adoption of advanced modulation formats [26].

Another device that can be adopted as reflective transmitter at the ONU side is the Reflective Electro-Absorption Modulator (REAM). EAM are semiconductor devices usually made in the form of a waveguide with electrodes for applying an electric field in a direction perpendicular to the modulated light beam. Their principle of operation is based on a change in the absorption spectrum caused by the applied electric field, which changes the band-gap energy without involving the excitation of carriers by the electric field: the so called Franz-Keldysh effect [27]. Reflective EAM include an EAM and a mirror, as schematically depicted in Figure 8, and they can be used to reflect and modulate the incoming light by means of the applied electrical signal $\left(V_{b}\right)$. Thanks to EAM, a modulation bandwidth close to $10 \mathrm{GHz}$ can be achieved, as shown in 
Figure 9, which makes these devices useful for optical fiber communication at $10 \mathrm{Gbit} / \mathrm{s}$ and above.

Differently from the RSOA, these devices do not amplify the light but, if coupled in cascade to a Semiconductor Optical Amplifier (SOA), they represent a very interesting solution for the ONU of a reflective PON architecture, since they provide a high-speed modulation capability, combined with the linear amplification of signal provided by the SOA. This solution has been proposed in several works, like for example [28, 29], in order to achieve a $10 \mathrm{Gbit} / \mathrm{s}$ upstream transmission in a WDM-PON scenario.

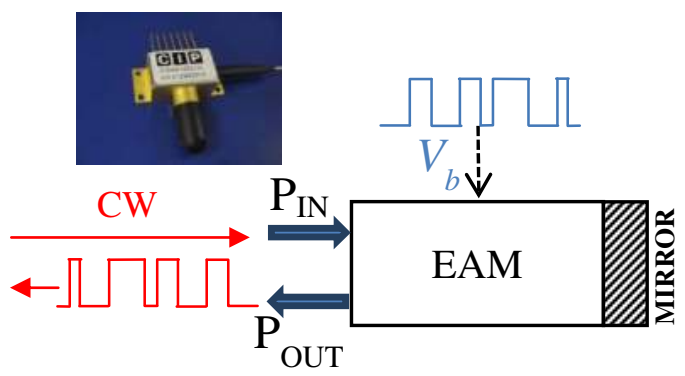

Figure 8. Schematic view of a REAM

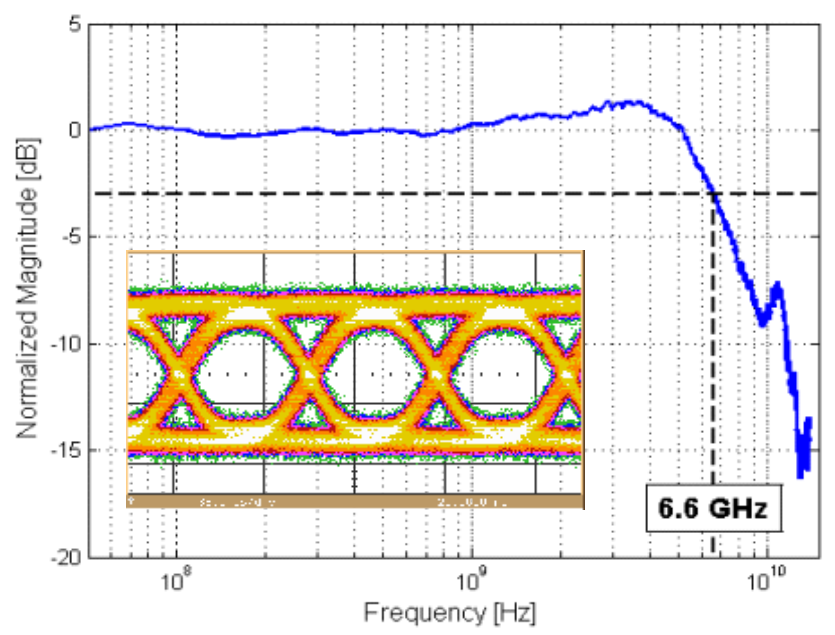

Figure 9. Characterization of a REAM

At the time of writing this Chapter, another solution for the reflective transmitter implementation is under analysis inside the FP7 EU STREP project titled "FABULOUS" [30]. It consists on a Mach-Zehnder based subsystem allowing polarization independent reflective carrier 
suppressed optical modulation for application in frequency division multiple access (FDMA) PON. The architecture of this subsystem, presented at first in [31], relies on using a MachZehnder modulator (MZM) simultaneously in both directions within a polarization diversity loop made of a polarization beam splitter (PBS) looped on itself through the modulator. The MZM provides RF access to both the electrodes independently, allowing modulation to be efficient in both the forward and the backward directions. On one electrode of the MZM, RF drive power is applied in the forward direction while, on the other electrode, the modulation is applied in the backward direction (the same signal is applied to both sides). The two polarizations of the incoming optical signal are split through the PBS and sent to the MZM in counter-propagating directions (the MZM having polarization maintaining fibers on both the input and the output). After being modulated, they are recombined through the PBS and sent back towards the OLT, as schematically depicted in Figure 10.

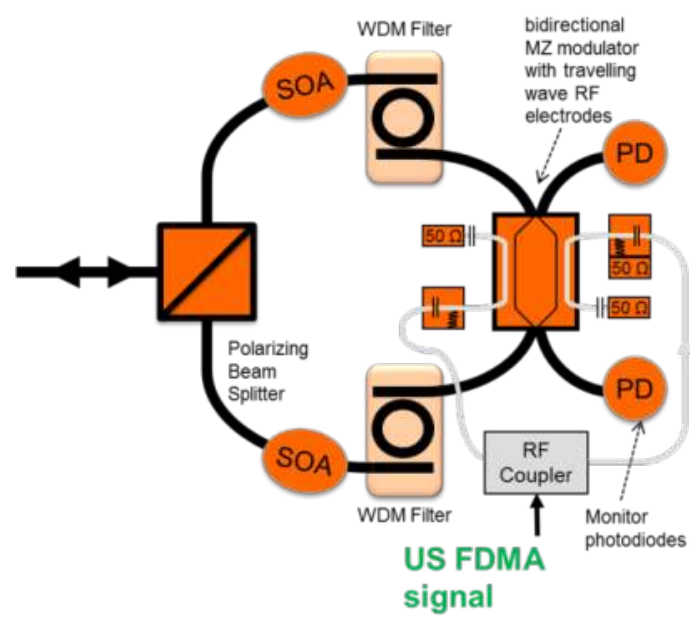

Figure 10. Architecture of R-MZM subsystem

\subsection{Transmission issues in reflective $P O N$}

The reflective WDM-PON architectures proposed in literature in the last ten years seem to be completely incompatible with TWDM-PON for a set of different reasons such as:

- most of them require the use of AWGs in the ODN, while as previously mentioned most telecom operators believes that backward compatibility in PON is a must, and thus the splitter-based architecture should be maintained also in NG-PON2;

- the achievable ODN loss is limited to typically 20-25 dB, due to several spurious effects such as the Rayleigh back-scattering and the low received optical power;

- most of them use a dedicated wavelength for each ONU, and do not support burst-mode TDMA in the upstream. 
In a reflective architecture like the one depicted in Figure 5, the upstream signal undergoes the ODN path loss twice (from the OLT to the ONU as CW seed signal and back to the OLT after the ONU reflection). The optical power at the OLT input is thus determined by the following formula:

$$
S=\vec{P}_{C W}-2 L{ }_{O D N}+G_{R O N U}
$$

where $\vec{P}_{C W}$ is the optical power of the CW signal at the input of the fiber, $L$ ODN is the ODN loss and $G_{R O N U}$ is the gain of the optical amplifier installed at the ONU. Since the TWDM-PON standard fixed the maximum value for the launched optical power at the ODN input to +11 $\mathrm{dBm}$ per wavelength and the gain of the optical amplifier at the ONU is typically of the order of $G_{R O N U}=20 \mathrm{~dB}$, the optical power at the OLT input, for the lowest ODN class N1 $\left(L_{O D N}=29 \mathrm{~dB}\right)$, is of the order of $S=-27 \mathrm{dBm}$, which is lower than the sensitivity of the standard direct-detection receivers. Moreover, it is well known that, in such an architecture, the spurious back reflections for a SMF fiber are of the order of 30-35 dB below the forward propagating signal, due to the concentrated reflections on components and the Rayleigh backscattering.

Rayleigh backscattering is an unavoidable phenomenon in optical fiber propagation. It is a fundamental loss mechanism arising from random density fluctuations frozen in the fiber during manufacturing. There is a growing interest in understanding Rayleigh backscattering since it can be a limiting factor in various optical systems. It must be taken into account in the performance computation of bidirectional lightwave system, especially in a wavelength-reuse system, like for example the reflective PON architecture depicted in Figure 11.

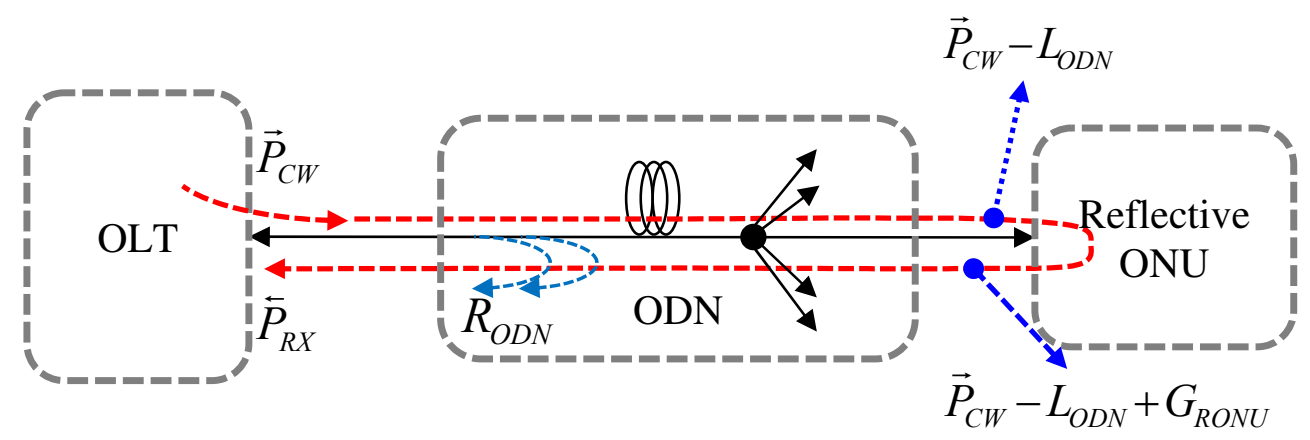

Figure 11. Rayleigh backscattering effect in reflective PON architecture at the OLT

In such a system, the received optical power is composed by the useful signal $S$, as specified above, and the interference signal I due to reflections, given by:

$$
I=\vec{P}_{C W}-R_{O D N}
$$


where $R_{O D N}$ is the power of the reflections. Therefore, the signal to interference ratio is given by:

$$
\left(\frac{S}{I}\right)_{d B}=-2 L_{O D N}+G_{R O N U}+R_{O D N}
$$

For a standard direct-detection receiver, even if the best solutions proposed in literature to mitigate the Rayleigh backscattering effect are adopted, the signal to interference ratio should be greater than 5-10 dB. This sets an important limit to the maximum achievable ODN loss. Indeed, the maximum reachable ODN loss is lower than $25 \mathrm{~dB}$, since the optical power of the spurious back reflections is about $30-35 \mathrm{~dB}$ below the forward propagating signal.

To improve the performance, one could in principle increase the $G_{R O N U}$, but there are technological component issues that limit the maximum reachable gain of optical amplifiers; in addition, another issue can arise from the Rayleigh backscattering that is generated at the ONU side, as depicted in Figure 12 and explained in details in [32]. This means that, in order to satisfy the NG-PON2 requirements with a reflective PON architecture, direct-detection at the OLT side is out of question.

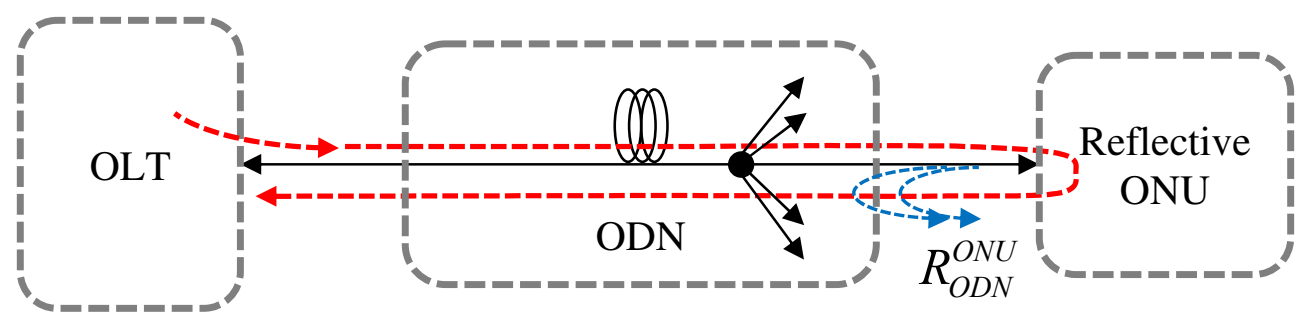

Figure 12. Rayleigh backscattering effect in reflective PON architecture at the ONU

\subsection{Self-coherent detection in RPONs}

Because of the aforementioned problems related to direct-detection, a coherent detection at the OLT seems to be a must in order to satisfy the NG-PON2 requirements, whether a reflective architecture approach is adopted.

The fundamental concept behind coherent detection is to use the product of the electrical fields of the modulated signal light (centred at $f_{S}$ ) and a CW local oscillator (centred at $f_{L O}$ ). This produces a frequency down-conversion of the signal to the frequency $f_{I F}=\left|f_{S}-f_{L O}\right|$, as schematically depicted in Figure 13.

A possible implementation of coherent detection in a reflective PON architecture is depicted in Figure 14: in this case, the CW light source placed at the OLT side is used both as a feed to be sent downstream to the reflective ONU and as a local oscillator for the OLT coherent 
(a)

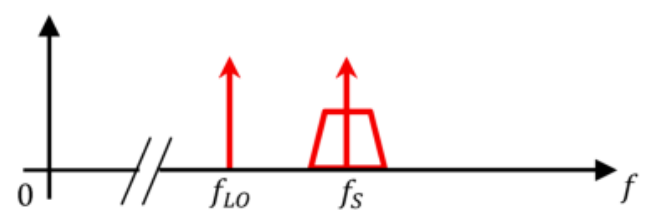

(b)

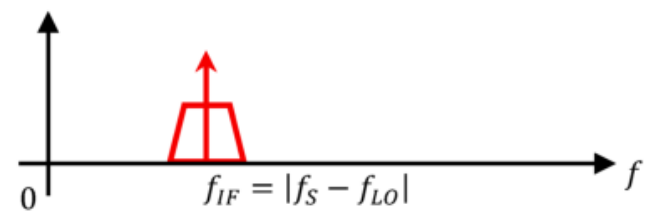

Figure 13. Spectra of (a) the optical signal and (b) the down-converted IF signal

receiver, executing a self-coherent detection. Therefore, after the optical-to-electrical conversion, the signal is down-converted to baseband.

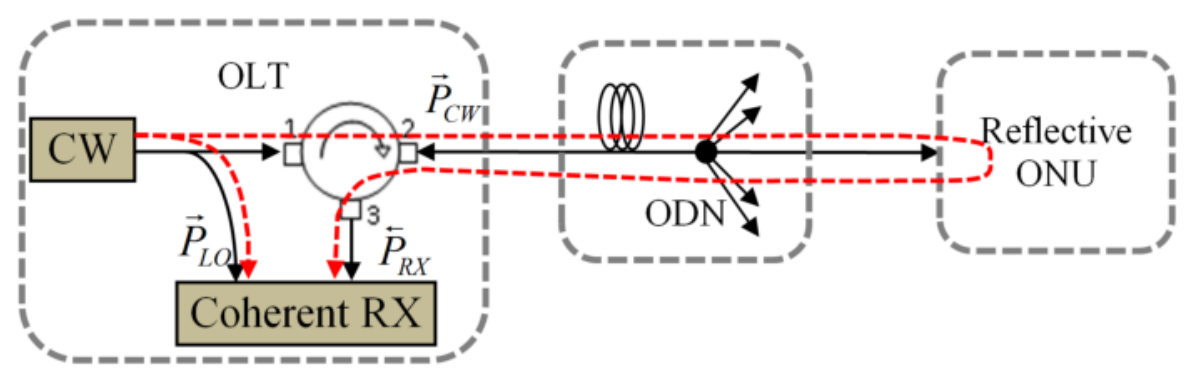

Figure 14. Scheme of a possible solution for self-coherent OLT in a reflective-PON architecture

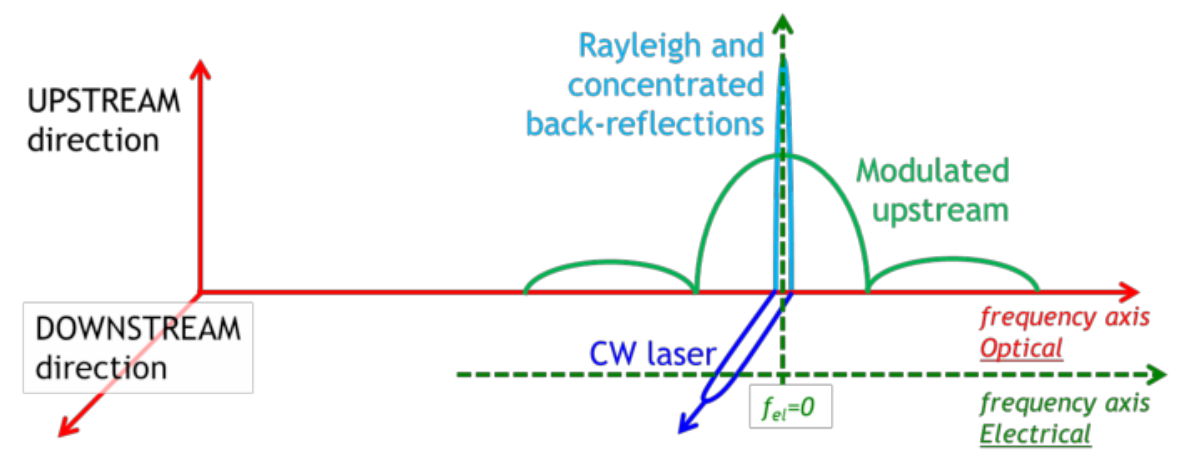

Figure 15. Spectral analysis of signal and reflections in reflective PON 
As demonstrated by the results available in literature presented in the following, this setup supports higher ODN loss values with respect to the limit of reflective PONs based on directdetection, in particular for the following reasons:

- much better sensitivity than direct-detection;

- much larger resilience to spurious back reflections.

Indeed, using a coherent receiver, the Rayleigh backscattering reflections appear as added close to the "electrical" DC, as depicted in Figure 15, thus they can be easily filtered out by electrical high-pass filters.

\section{Latest research results}

WDM reflective PON "variant", characterized by self-coherent detection at the OLT side, was initially proposed in [33], where a RSOA-based WDM PON architecture, employing a novel self-homodyne receiver and a novel signal processing technique to eliminate the penalty caused by the back-reflection of the seed light, is proposed. In addition, they successfully demonstrated a long-reach fiber-loopback system of over $100 \mathrm{~km}$. These results indicate that the proposed architecture could provide an attractive solution to realize a long-reach WDM PON with high-split ratio.

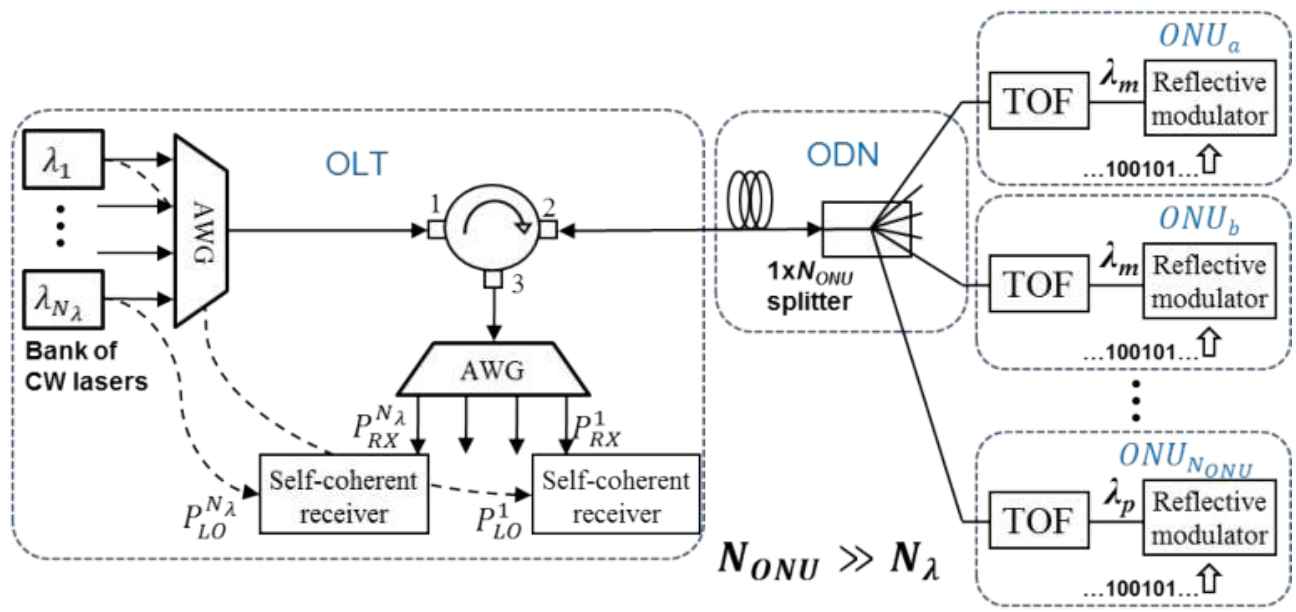

Figure 16. Scheme of self-coherent reflective PON architecture (only upstream transmission) 
A possible implementation of the upstream transmission in a self-coherent reflective PON is shown in Figure 16; this architecture may offer the following advantages:

- the upstream wavelengths comb accuracy is completely set by the OLT and not by each individual ONU. In this solution, each ONU needs to tune its optical filter by locking it on one of the $N_{\lambda}$ already existing wavelength;

- subsequent upgrades to dense-WDM (DWDM) seem more feasible when using a number of wavelengths $N_{\lambda}$ significantly higher than 4 , and possibly a narrower frequency spacing (such as $50 \mathrm{GHz}$ ), since it is possible to avoid tunable lasers at the ONU;

- for each upstream wavelength, the required CW laser and coherent receiver at the OLT are shared by several ONUs (of the order of $N_{O N U} / N_{\lambda}$ ) so that their cost may be more reasonably justified.

In addition to WDM, a second level of multiplexing is necessary in order to overcome the limit of a dedicated wavelength per user; this can be obtained sharing time, basing on Time Division Multiplexing (TDM) in the downstream and Time Division Multiple Access (TDMA) in the upstream, or frequency (FDM and FDMA) between users.

\subsection{TDM-WDM results}

Basing on the architecture depicted in Figure 16, the results presented in [34] experimentally demonstrate the simultaneous transmission of two independent TDMA ONUs with upstream bit rate equal to $2.5 \mathrm{Gbit} / \mathrm{s}$, working in TDMA burst mode and with performance compatible with E2 XG-PON class, thus compatible with US TWDM-PON requirements. At the OLT, the signal demodulation is performed thanks to a self-coherent receiver and a custom burst-mode digital-signal processing technique. In particular, a conventional DSP solution based on Viterbi-Viterbi carrier-phase estimation and LMS adaptive equalization [35, 36] has been modified to work in burst mode operation, focusing on the alignment procedures on the received packets and on the convergence speed of the LMS algorithm [37, 38].

The reflective ONU of such architecture is emulated as depicted in Figure 17. The CW signal reaching the ONU is reflected and modulated by means of a REAM. The SOA placed in front of the REAM amplifies the optical signal twice, first on the feed CW downstream, and then on the reflected and modulated upstream signal. In order to emulate the ONU wavelength selection functionality, a TOF is placed between the SOA and the REAM. This TOF is also useful to partially filter out the ASE noise and, thanks to its approximately $4 \mathrm{~dB}$ insertion loss, to avoid excessive SOA saturation in the upstream direction. The SOA+REAM structure, even though not yet commercial, has been integrated in several research projects (such as in [40]). The $2.5 \mathrm{Gbit} / \mathrm{s}$ upstream signal is generated at the ONU by driving the REAM by pure NRZ signal, while the optical packets are generated by switching on and off the SOA.

Each packet contains a short dummy-pattern at the beginning and end of each burst, 127 bits of sync-pattern and 1000 bits payload (8B/10B coded), as shown in Figure 18. The dummypattern is useful for "absorbing" the rise and fall-time of the SOA acting as a gate, and also the transient effect of the coherent receiver AC-coupling. The sync-pattern is used for identifying 


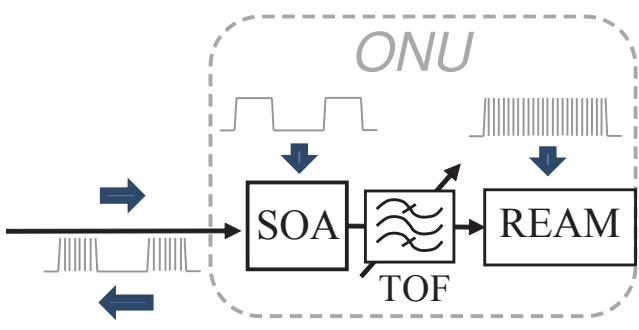

Figure 17. Reflective ONU implementation

the start of each packet and also for training the LMS algorithm, before switching to the LMS "decision-directed" mode, which should carry out the burst payload elaboration. This system emulates a TDMA transmission from two independent ONUs with 25 ns guard-time, where the ONU 1 is the reference ONU and the ONU 2 is the interfering ONU, reproducing the worstcase condition in terms of interference for such a system.

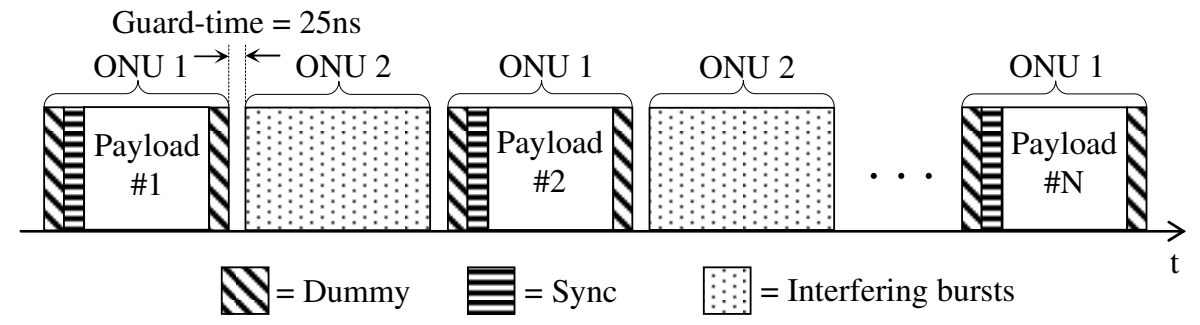

Figure 18. Time relations of the useful and interfering optical US packets for two ONUs

The authors of [34] expressed the performance of the system in terms of Bit Error Rate (BER) as a function of the ODN loss and the acceptable differential optical path loss (DOPL).

The results show that a BER $<10^{-3}$ (a reasonable Forward Error Correcting code threshold for simple FECs, as those used in XG-PON) is obtained up to $35 \mathrm{~dB}$ of ODN loss and more than $15 \mathrm{~dB}$ of DOPL, as required by XG-PON class E2.

The same setup was also tested increasing the upstream bit rate and using commercial DFB lasers rather than ECL lasers at the OLT side [39]. The bit rate was set to $10 \mathrm{Gbit} / \mathrm{s}$ per wavelength (from the previous $2.5 \mathrm{Gbit} / \mathrm{s}$ rate), a bit rate currently under consideration in FSAN for the point-to-point WDM (PtP-WDM) for symmetric traffic, and for the TWDMPON longer term evolution. Replacing the ECL lasers with DFB lasers would provide a significant cost reduction at the OLT. As reported in [39], in these working conditions, the FEC threshold is reached after $31 \mathrm{~dB}$ and for a DOPL higher than $15 \mathrm{~dB}$, satisfying the requirement of $\mathrm{N} 2$ class. 


\subsection{FDM-WDM results}

One of the main drawbacks of the more traditional TDMA-PON approach deployed nowadays, is that it does not scale well above $10 \mathrm{Gbit} / \mathrm{s}$ per wavelength in term of cost/complexity and power efficiency, mostly due to the fact that each ONU should work on the aggregated bit rate.

On the contrary, FDM/FDMA approach allows ONUs to operate at low DSP speeds (and so reduce their cost and power consumption); indeed, the speed at which the ONUs operates is equal to the maximum speed that the customers are allowed to communicate at (e.g. $1 \mathrm{Gbit} / \mathrm{s})$ which is much smaller than the aggregated line rate (e.g. $20 \mathrm{Gbit} / \mathrm{s})$.

The work presented in [41] proposes an innovative reflective PON approach for the upstream transmission using a special configuration based on FDMA, where each ONU is assigned a portion of the available electrical spectrum to perform an high spectral efficiency M-QAM modulation format as depicted in Figure 19.

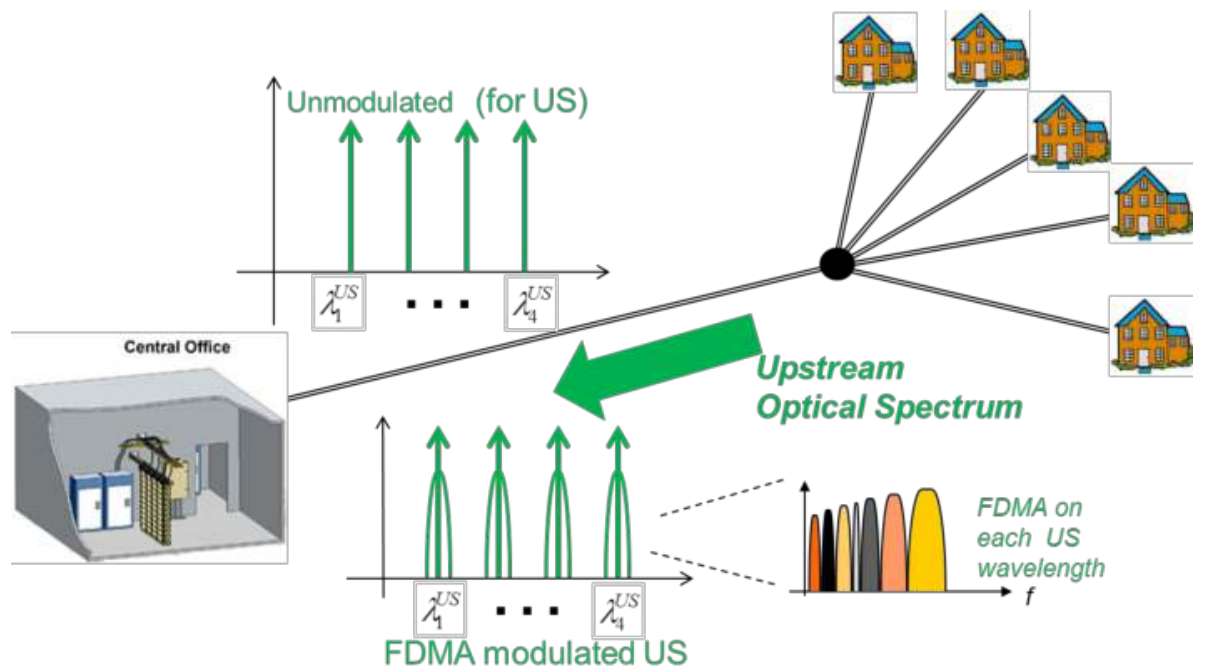

Figure 19. FDMA reflective PON architecture

The results presented in [41] demonstrate that this architecture targets an upstream capacity of $32 \mathrm{Gbit} / \mathrm{s}$ per optical carrier, outperforming NG-PON2 with a tenfold increase in the upstream capacity, with power budget and max reach overcoming ITU-T class N2, as defined in the XG-PON standard (up to $40 \mathrm{~km}$ and $31 \mathrm{~dB}$ ODN loss). An important feature of this approach consists in the fact that, due to the absence of optical sources at the customer premises, the ONU can be realized as a Photonic Integrated Circuit with an unprecedented level of integration. This holds true for self-coherent reflective PONs in general, but the highest level of integration is to date relevant to the architecture shown in [41], where the Mach 
Zehnder Modulator, the two tunable filters (for multi-wavelength transmission) and the polarizing beam splitter that characterize the proposed ONU will be realized on silicon platform, the two SOA will be suitable for photonic integration and finally the electrical driver will be flip-chipped on top; for a detailed description see [42, 43].

\section{Conclusions}

In this Chapter, we have given a general description of the self-coherent reflective PON architecture as a possible technological approach to the NG-PON2 requirements. We have shown that, thanks to reflective ONU and the self-coherent detection at the OLT side, the performance required by NG-PON2 are satisfied even without the need for a tunable laser at the ONU side. If properly integrated, the reflective ONU structure can be less expensive than the TWDM-PON solution in term of CAPEX, and have a much simpler wavelength control. The only significant increase in cost in the proposed solution is due to the presence of one coherent receiver per each upstream wavelength (a cost to be shared among all the users with the same wavelength). While the cost of coherent receivers used today for long-haul is likely still too high for an OLT, it can be observed an enormous effort in the long-haul technical community to make small-form factor and low-power coherent receivers possible, so that it is reasonable to envision a sharp decrease in coherent receiver costs that may allow the use of coherent detection at OLT in the near term.

\section{Author details}

S. Straullu ${ }^{1 *}$, S. Abrate ${ }^{1}$ and R. Gaudino ${ }^{2}$

*Address all correspondence to: straullu@ismb.it

1 Istituto Superiore Mario Boella, Torino, Italy

2 Politecnico di Torino, Torino, Italy

\section{References}

[1] E. Harstead, et al., “Technologies for NGPON2: Why I think 40G TDM PON (XLGPON) is the clear winner", in Proc. Opt. Fiber Commun. Conf./Nat. Fiber Opt. Eng. Conf. Workshop, Mar. 2012

[2] X. Hu, et al., "Energy-efficient WDM-OFDM-PON employing shared OFDM modulation modules in optical line terminal", Opt. Exp., vol. 20, no. 7, pp. 8071-8077, Mar. 2012. 
[3] W. Wei et al., "Optical orthogonal frequency division multiple access networking for the future internet", IEEE/OSA J. Opt. Commun. Netw., vol. 1, no. 2, pp. 236-246, Jul. 2009.

[4] H. K. Lee et al., "A WDM-PON with an $80 \mathrm{~Gb} / \mathrm{s}$ capacity based on wavelengthlocked Fabry-Perot laser diode", Opt. Exp., vol. 18, no. 17, pp. 18077-18085, Aug. 2010.

[5] K. Grobe et al., "PON in adolescence: from TDMA to WDM-PON", IEEE Commun. Mag., vol. 46, no. 1, pp. 26-34, Jan. 2008.

[6] Y. Luo at al., "Time-and Wavelength-Division Multiplexed Passive Optical Network (TWDM-PON) for Next-Generation PON Stage 2 (NG-PON2)", IEEE J. LIGHTWAVE TECH. 31 no. 4, 587-593 (2013).

[7] M. Zhu et al., “Upstream multi-wavelength shared PON with wavelength-tunable self-seeding Fabry-Perot laser diode," in Proc. Opt. Fiber Commun. Conf./Nat. Fiber Opt. Eng. Conf., Mar. 2011, pp. 1-3.

[8] D. Liang et al., "Low threshold electrically-pumped hybrid silicon microring lasers," IEEE J. Sel. Topics Quantum Electron., vol. 17, no. 6, pp. 1528-1533, Nov./Dec. 2011.

[9] A. Kato et al., "Tunable optical filter with cascaded waveguide Fabry-Pérot resonators featuring liquid crystal cladding," Photon. Technol. Lett., vol. 24, no. 4, pp. 282 284, Feb. 2012.

[10] R. Murano et al., "Tunable $2.5 \mathrm{~Gb} / \mathrm{s}$ receiver for wavelength-agile DWDM-PON," presented at the presented at the Opt. Fiber Commun. Conf./Nat. Fiber Opt. Eng. Conf., 2008, Post Deadline Paper.

[11] G. W. Yoffe et al., "Widely-tunable $30 \mathrm{~mW}$ laser source with sub-500 kHz linewidth using DFB array," in Proc. IEEE Lasers Electro-Optics Soc., Nov. 2008, pp. 892-893.

[12] D. Ton et al., "2.5-Gb/s modulated widely tunable laser using an electroabsorption modulated DFB array and MEMS selection," IEEE Photon. Technol. Lett., vol. 16, no. 6, pp. 1573-1575, Jun. 2004.

[13] Y. Liu et al., "Uncooled DBR laser directly modulated at $3.125 \mathrm{~Gb} / \mathrm{s}$ as athermal transmitter for low-cost WDM systems," IEEE Photon. Technol. Lett., vol. 17, no. 10, pp. 2026-2028, Oct. 2005.

[14] P. Ossieur et al.,“Demonstration of a 32512 Split, 100 km Reach, 23210 Gb/s hybrid DWDM-TDMA PON using tunable external cavity lasers in the ONUs," J. Lightw. Technol., vol. 29, no. 24, pp. 3705-3718, Dec. 2011.

[15] F. Wei et al. "Tunable external cavity diode laser with a PLZT electrooptic ceramic deflector," IEEE Photon. Technol. Lett., vol. 23, no. 5, pp. 296-298, Mar. 2011.

[16] J. Zheng et al., "Optically tunable ring external-cavity laser," in Proc. IEEE Photon. Conf., Oct. 2011, pp. 644-645. 
[17] http://www.freepatentsonline.com/7046435.html

[18] P. Healey et. al., "Spectral slicing WDM-PON using wavelength-seeded reflective SOAs", Electronics Letters, 37, 19, 1181 - 1182 (2001).

[19] H.C. Shin et al., "Reflective SOAs optimized for 1.25 Gbit/s WDM-PONs", Lasers and Electro-Optics Society (LEOS), Vol. 1 (2004).

[20] L. Wooram et al., "Bidirectional WDM-PON based on gain-saturated reflective semiconductor optical amplifiers", Photonics Technology Letters, IEEE, 17, 11, 2460 - 2462 (2005).

[21] C. Arellano et al, "Bidirectional single fiber transmission based on a RSOA ONU for FTTH using FSK-IM modulation formats", Optical Fiber Communication Conference, 2005, OFC/NFOEC, Vol. 3 (2005).

[22] E. Wong et al., "Low-cost WDM passive optical network with directly-modulated self-seeding reflective SOA", Electronics Letters, 42, 5, 299 - 301 (2006).

[23] I. Papagiannakis et al., "Investigation of 10-Gb/s RSOA-Based Upstream Transmission in WDM-PONs Utilizing Optical Filtering and Electronic Equalization", Photonics Technology Letters, IEEE, 20, 24, 2168 - 2170 (2008).

[24] K.Y. Cho et al., "10-Gb/s Operation of RSOA for WDM PON", Photonics Technology Letters, IEEE, 20, 18, 1533 - 1535 (2008).ù

[25] G. Qi et al., "Low-Bandwidth RSOA Using Partial Response Equalization", Photonics Technology Letters, IEEE, 23, 20, 1442 - 1444 (2011).

[26] T. Duong et al., "Experimental demonstration of $10 \mathrm{Gbit} / \mathrm{s}$ upstream transmission by remote modulation of $1 \mathrm{GHz}$ RSOA using Adaptively Modulated Optical OFDM for WDM-PON single fiber architecture", ECOC 2008.

[27] http://users.df.uba.ar/bragas/Web roberto/Papers/Ralph Franz Keldish.pdf

[28] E.K. MacHale et al., "Extended-Reach PON Employing 10Gb/s Integrated Reflective EAM-SOA", ECOC 2008.

[29] D. Smith et al., "Colourless 10Gb/s reflective SOA-EAM with low polarization sensitivity for long-reach DWDM-PON networks", ECOC 2009.

[30] http://www.fabulous-project.eu/

[31] B. Charbonnier et al., "Reflective polarisation independent Mach-Zenhder modulator for FDMA/OFDMA PON", Electronics Letters, 46, 25, 1682 - 1683 (2010).

[32] C. Arellano et al., "Reflections and Multiple Rayleigh Backscattering in WDM SingleFiber Loopback Access Networks", IEEE J. LIGHTWAVE TECH. 27 no. 1, 12-18 (2009). 
[33] S.P. Jung et al., "Demonstration of RSOA-based WDM PON employing self-homodyne receiver with high reflection tolerance", Optical Fiber Communication Conference, OFC/NFOEC (2009).

[34] S. Straullu et al., "Compatibility between coherent reflective burst-mode PON and TWDM-PON physical layers", OPEX 22, Issue 1, pp. 9-14 (2014), http://dx.doi.org/ 10.1364/OE.22.000009

[35] K. Y. Cho et al., "10-Gb/s operation of RSOA for WDM PON," IEEE PHOTON. TECHNOL. LETTER, 20, 18, 1533 - 1535 (2008).

[36] S. J. Savory, "Digital filters for coherent optical receivers," OPEX 16, 2, 804-817 (2008).

[37] F. Vacondio et al., "Experimental demonstration of a PDM QPSK real-time burst mode coherent receiver in a packet switched network," ECOC, Tu.3.A.1, (2012).

[38] F. Vacondio, et al. "Flexible TDMA Access Optical Networks Enabled by Burst-Mode Software Defined Coherent Transponders", ECOC, We.1.F.2, London, UK, (2013).

[39] S. Straullu et al., “TWDM-PON-compatible 10 Gbps Burst-mode coherent reflective ONU achieving $31 \mathrm{~dB}$ ODN loss using DFB lasers", ECOC, Cannes, France (2014).

[40] A. Naughton et al., “Optimisation of SOA-REAMs for hybrid DWDM-TDMA PON applications", Optics Express, 19, 26 (2011).

[41] S. Straullu et al., "Reflective FDMA-PON with 32 Gbps upstream capacity per wavelength and more than 32 dB ODN loss", ECOC, Cannes, France (2014).

[42] S. Abrate et. al., "FDMA-PON architecture according to the FABULOUS European Project" Proc. SPIE 8645, Broadband Access Communication Technologies VII, 864504 (2013).

[43] S. Menezo et al., "Reflective silicon Mach-Zehnder modulator with Faraday rotator mirror effect for self-coherent transmission", OFC/NFOEC 2013. 